\title{
Beta-blockers for heart failure
}

\author{
John GF Cleland ${ }^{1}$, Nick Freemantle ${ }^{2}$, Joanne Eastaugh ${ }^{3}$, Phillip J Young ${ }^{4}$, and Jane \\ Harrison ${ }^{3}$ \\ ${ }^{1}$ Academic Unit of Cardiology, Castle Hill Hospital, East Yorkshire, UK \\ ${ }^{2}$ Department of Primary Care and General Practice, University of Birmingham, Birmingham, UK \\ ${ }^{3}$ Medicines Evaluation Group, Centre for Health, University of York, York, UK \\ ${ }^{4}$ Health Sciences and Clinical Evaluation, University of York, York, UK
}

\begin{abstract}
This is the protocol for a review and there is no abstract. The objectives are as follows:

Our objective is to appraise the effectiveness of beta blockers in patients with heart failure. Our protocol defined main outcome is all cause mortality. The specific a priori defined aims are to examine: the effectiveness of beta blockers in all trials of patients with heart failure, and examine the importance of the presence or absence of ischaemic cardiomyopathy in patients included in trials and vasodilator properties of beta blocking agents used. We will also examine the predictive value of left ventricular function, age, use of angiotensin converting enzyme inhibitors and New York Heart Association Class (NYHA), and the rate of discontinuation of therapy due to treatment.
\end{abstract}

There are a number of important ongoing trials for which data will become available in the next few years. Thus a systematic review which may be updated regularly is required to provide an up to date synthesis of the available data in this increasingly important area.

\section{BACKGROUND}

There is considerable interest in the use of beta blockers in patients with heart failure. For many years such an application of beta blockers was considered counter intuitive, and many trials of beta blockers for secondary prevention explicitly excluded patients with signs of

Copyright () 2009 The Cochrane Collaboration. Published by John Wiley \& Sons, Ltd.

Contact address: John GF Cleland, Academic Unit of Cardiology, Castle Hill Hospital, Castle Road, Cottingham, East Yorkshire, HU16 5JQ, UK. j.g.cleland@ hull.ac.uk.j.g.cleland@medschool.hull.ac.uk.

Editorial group: Cochrane Heart Group.

Publication status and date: Edited (no change to conclusions), published in Issue 1, 2009.

CONTRIBUTIONS OF AUTHORS

John Cleland - protocol development and intermediate results

Nick Freemantle - data extraction and meta-analysis

Joanne Eastaugh - data extraction

Phil Young - statistical advice

Jane Harrison - literature search

DECLARATIONS OF INTEREST

Nick Freemantle received an unrestricted research grant from

SmithKline Beecham, who market Carvedilol, a Beta Blocker, in the US. 
heart failure. However, recent systematic reviews of the efficacy of beta blockers in heart failure have identified a number of relevant randomised trials which were suggestive of a beneficial effect from beta blockade in this patient group (Lechat 1998; Doughty 1997; Heidenreich 1997).

The development of regression techniques within meta analysis enables the more robust examination of the importance of factors that may mediate upon the effectiveness of specific drugs (Smith 1995). For instance, there is a suggestion in the literature that beta blockers with intrinsic sympathomimetic activity may be less efficacious in reducing mortality than beta blockers without this action (Freemantle 1999).

\section{OBJECTIVES}

Our objective is to appraise the effectiveness of beta blockers in patients with heart failure. Our protocol defined main outcome is all cause mortality. The specific a priori defined aims are to examine: the effectiveness of beta blockers in all trials of patients with heart failure, and examine the importance of the presence or absence of ischaemic cardiomyopathy in patients included in trials and vasodilator properties of beta blocking agents used. We will also examine the predictive value of left ventricular function, age, use of angiotensin converting enzyme inhibitors and New York Heart Association Class (NYHA), and the rate of discontinuation of therapy due to treatment.

There are a number of important ongoing trials for which data will become available in the next few years. Thus a systematic review which may be updated regularly is required to provide an up to date synthesis of the available data in this increasingly important area.

\section{METHODS}

\section{Criteria for considering studies for this review}

Types of studies-Adequately randomised trials comparing beta blockers with control in patients with evidence of heart failure.

Types of participants-Patients with symptomatic heart failure (NYHA > I), who may or may not have experienced a myocardial infarction, randomised to receive treatment with beta blockers or control, with at least 1 month duration.

\section{Types of interventions-}

1. Oral beta blockade in any dose

2. Placebo or no treatment control

Types of outcome measures-All cause mortality is consistently reported in trials, and given the large number of patients already randomised it will provide the best overall estimate of treatment effect. A recent review (Lechat 1998) has indicated that NYHA functional class and number of hospitalisations may be improved by beta blockade and will therefore also be included. In later revisions of this review we will consider inclusion of appropriate additional outcomes as these may be useful in achieving a greater understanding 
of the nature of any treatment effect identified. We will also include the rate of withdrawal from therapy as this will indicate the proportion of patients who may tolerate beta blockade.

\section{Search methods for identification of studies}

We will conduct sensitive electronic searches of MEDLINE (1966-97 via OVID), EMBASE (1974-97 via DIALOG), Biosis (1985-97 via EDINA), Healthstar (1975-97 via OVID), Sigle (1980-1997 via BLAISE-LINE), IHTA (1990-1997 via ECRInet), Conference Papers Index (1984-1997 via DIAL0G), Derwent Drug File (1992-1997 via DIAL0G), Dissertation Abstracts (1992-1997 via DIAL0G), Pascal (1992-1997 via DIAL0G), International Pharmaceutical Abstracts (1992-1997 via DIAL0G) and Science Citation Index (1981-1997 via BIDS).

The reference list of each identified study will be reviewed. We will also examine existing bibliographies and reviews for relevant studies.

Searches will be performed by an experienced member of staff of the NHS Centre for Reviews and Dissemination. Searches including the Cochrane CENTRAL Trials Registry and the McMaster CVD Trials Registry will be considered for future updates of the review.

\section{Data collection and analysis}

Data synthesis-Data on the total number of patients randomised to active treatment or control, beta blocker, route and dose, duration of treatment, loss to follow up, level of blinding, concealment of allocation (Schultz 1995), specific study inclusion and exclusion criteria, duration of follow up and deaths will be abstracted from each study. Abstracted data will be checked by a second researcher.

We will estimate pooled odds ratios for short and long term treatment trials separately using the fixed effects approach of Mantel Haenszel (Rothman 1986; Robins 1986). Since a priori we anticipate systematic differences between the results of studies (heterogeneity) we will also routinely estimate random effects pooled odds ratios using the approach described by Fleiss \& Gross (Fleiss 1991), itself based upon the methods of DerSimonian \& Laird (DerSimonian 1986). Risk differences will be calculated using standard random effects methods (DerSimonian 1986). Because comparison of risk differences between trials may be confounded by different lengths of follow up, we will estimate a pooled incidence risk difference using the approach described by Ioannidis et al (Ioannidis 1995). We will also calculate pooled estimates of effect for each individual beta blocker using the fixed effects model (Rothman 1986; Robins 1986).

Standard random effects methods for meta analysis (pooling the results of studies) may be biased as they assume that the observed distribution of effects is the true treatment distribution, an assumption that may not be valid in sparse data, (Smith 1995; Hardy 1996; Carlin 1992) or where there are a substantial number of trials that have no events in either the intervention or control groups, or even both. Therefore we will use the full random effects approach, based upon Markov chain Monte Carlo numerical integration techniques, using appropriate uninformative priors (Smith 1995), using the 'BUGS' software described in that paper. 
A further advantage of this approach is that the effects of covariates may be examined. Our main protocol defined treatment related covariates are presence or absence of ischaemic cardiomyopathy in patients included in trials and vasodilator properties of beta blocking agents used, and the choice of drug. We will also examine the predictive value of left ventricular function, age, use of angiotensin converting enzyme inhibitors, proportion of patients who had experienced a MI prior to randomisation and New York Heart Association Class which will be examined using the a random effects logistic regression model.

Convergence will be assessed using the methods described by Geweke (Geweke 1992), and visual inspection of convergence plots.

\section{Acknowledgments}

Internal sources

- University of Hull, UK.

- University of York, UK.

External sources

- $\quad$ No sources of support supplied

\section{WHAT'S NEW}

\begin{tabular}{lll}
\hline Date & Event & Description \\
\hline 8 September 2008 & Amended & Converted to new review format. \\
\hline
\end{tabular}

\section{HISTORY}

Protocol first published: Issue 2, 2000

\section{Additional references}

Carlin 1992 . Carlin JB. Meta analysis for $2 \times 2$ tables: A Bayesian approach. Stats in Med. 1992; 11:141-58. MEDLINE: 92253885;: ISSN 02776715.

DerSimonian 1986 . DerSimonian R, Laird N. Meta-analysis in clinical trials. Controlled Clin Trials. 1986; 7:177-88. MEDLINE: 87104256;: ISSN 01972456. [PubMed: 3802833]

Doughty 1997 . Doughty RN, Rodgers A, Sharpe N, MacMahon S. Effects of beta-blocker therapy on mortality in patients with heart failure. Eur Heart J. 1997; 18:560-65. MEDLINE: 97276130;: ISSN 0195668X. [PubMed: 9129883]

Fleiss 1991 . Fleiss J, Gross AJ. Meta-analysis in epidemiology, with special reference to studies of the association between exposure to environmental tobacco smoke and lung cancer: A critique. J Clin Epidemiol. 1991; 44:127-39. MEDLINE: 91140201;: ISSN 08954356. [PubMed: 1995774]

Freemantle 1999 . Freemantle N, Cleland JGF, Young P, Mason J, Harrison J. What is the place of beta-blocade in secondary prevention after myocardial infarction? A systmeatic review and meta regression analysis. BMJ. 1999; 318:1730-7. MEDLINE: 99310729;: ISSN 09598138. [PubMed: 10381708]

Geweke 1992 . Geweke, J. Evaluating the accuracy of sampling based approaches to calculating posterior moments. In: Bernardo, JM.; Berger, JO.; Dawid, AP.; Smith, AFM., editors. Bayesian Statistics. Vol. 4. Clarendon Press; Oxford: 1992.

Hardy 1996 . Hardy RJ, Thompson SG. A likelihood approach to meta analysis with random effects. Stats in Med. 1996; 15:619-29. MEDLINE: 96294125;: issn 02776715. 
Heidenreich 1997 . Heidenreich PA, Lee TT, Massie BM. Effect of beta-blockade on mortality in patients with heart failure: a meta analysis of randomized clinical trials. J Am Coll Cardiol. 1997; 30:27-34. MEDLINE: 97351335;: ISSN 07351097. [PubMed: 9207617]

Ioannidis 1995 . Ioannidis JPA, Cappelleri JC, Lau J, Skolnik PR, Melville B, Chalmers TC, Sacks HS. Early or deferred zidovudine therapy in HIV-infected patients without an AIDS defining illness. Ann Intern Med. 1995; 122:856-66. MEDLINE: 95259942;: ISSN 00034819. [PubMed: 7741372]

Lechat 1998 . Lechat P, Packer M, Chalon S, Cucherat M, Arab T, Boissel JP. Clinical effects of beta-adrenergic blockade in chronic heart failure: a meta-analysis of double-blind, placebocontrolled, randomized trials. Circulation. 1998; 98:1184-91. MEDLINE: 98416144;: 00097322. [PubMed: 9743509]

Robins 1986 . Robins J, Breslow N, Greenland S. Estimators of the Mantel-Haenszel variance consistent in both sparse data and large strata models. Biometrics. 1986; 42:311-23. MEDLINE: 86296961;: ISSN 0006341X. [PubMed: 3741973]

Rothman 1986 . Rothman, KJ. Modern Epidemiology. Little \& Brown; Boston: 1986.

Schultz 1995 . Schulz KF, Chalmers I, Hayes RJ, Altman DG. Empirical evidence of bias: dimensions of methodological quality associated with estimates of treatment effects in controlled trial treatment effects in controlled trials. JAMA. 1995; 273:408-12. [PubMed: 7823387]

Smith 1995 . Smith TC, Spiegelhalter DJ, Thomas A. Bayesian approaches to random-effects meta analysis: a comparative study. Stats in Med. 1995; 14:2685-99. MEDLINE: 96201053;: ISSN 92253885 .

\section{References to other published versions of this review}

Cleland 1999 . Cleland JGF, Freemantle N, McGowan J, Clark A. The evidence for Beta-Blockers equals that for ACE Inhibitors in Heart Failure. BMJ. 1999; 318:824-25. [PubMed: 10092240]

* Indicates the major publication for the study 